\title{
Quality of Life before and after the Use of Vaginal Estriol in Postmenopausal Women with Lower Urinary Tract Symptoms
}

\author{
Silvia Veiga Teixeira de Freitas*, Carlos Augusto Faria \\ School of Medicine, Universidade Federal Fluminense, Niterói, Brazil \\ Email: *silviavtfreitas@gmail.com
}

How to cite this paper: de Freitas, S.V.T. and Faria, C.A. (2020) Quality of Life before and after the Use of Vaginal Estriol in Postmenopausal Women with Lower Urinary Tract Symptoms. Open Journal of Obstetrics and Gynecology, 10, 452-462. https://doi.org/10.4236/ojog.2020.1040041

Received: March 10, 2020

Accepted: April 5, 2020

Published: April 8, 2020

Copyright () 2020 by author(s) and Scientific Research Publishing Inc. This work is licensed under the Creative Commons Attribution International License (CC BY 4.0).

http://creativecommons.org/licenses/by/4.0/

\begin{abstract}
Background: Nowadays, a substantial number of women spend more than one-third of their lives in the postmenopausal period. It is characterized by a marked decrease in the production of estrogen, which leads to urogenital atrophy. The symptoms stemming from vulvovaginal atrophy and the lower urinary tract are currently referred to as the Genitourinary Syndrome of Menopause (GSM), which can have a negative impact on the quality of life (QoL). Estrogen replacement is the ideal treatment for GSM, and vaginal administration is the most recommended. Objective: To assess the impact of the use of topical vaginal estriol on the quality of life (QoL) of postmenopausal women with lower urinary tract symptoms (LUTS). Methods: This is an interventional, prospective study, performed in 49 women at the Antônio Pedro Hospital, at the Universidade Federal Fluminense, in Niterói, Brazil, from August 2014 to April 2015. It included postmenopausal women with lower urinary tract symptoms who were not using any estrogen hormone therapy, and it excluded those with contraindications for the use of estriol. A specific questionnaire on QoL and urinary tract symptoms, the King's Health Questionnaire (KHQ), which identifies LUTS and assesses to what extent those symptoms interfere with QoL, has been used as a research tool. Results: The average age was 62.24 years. Urinary urgency and urge incontinence were reported by $91.8 \%$ of women. The average scores of the domains of the KHQ decreased in the domains General Health Perception (before: $46.42 \pm 21.65$; after: $40.81 \pm 22.64$ ), Incontinence Impact (before: $74.82 \pm 27.66$; after: 41.49 \pm 30.83 ), Role Limitations (before: $43.20 \pm 32.80$; after: $21.09 \pm 24.71$ ), Physical Limitations (before: $38.09 \pm 32.09$; after: $14.62 \pm 24.20$ ), Social Limitations (before: $30.38 \pm 28.75$; after: $12.62 \pm 19.85$ ), Emotions (before: $43.31 \pm 32.96$; after: $20.18 \pm 26.41$ ), Sleep/Energy (before: $41.48 \pm 37.74$; after: $15.98 \pm 23.31$ ) and Severity Measures (before: $48.02 \pm 24.68$; after: $22.31 \pm 20.25$ ). All the
\end{abstract}


differences were statistically significant (p-value $\leq 0.05$ ). Conclusion: In the group of postmenopausal women with lower urinary tract symptoms included in the study, the use of topical vaginal estriol led to a decrease in the frequency of each symptom and the average scores in all domains of the KHQ, suggesting a positive effect on women's QoL.

\section{Keywords}

Post-Menopause, Lower Urinary Tract Symptoms, Estriol, Quality of Life

\section{Introduction}

The increase in life expectancy experienced in recent years has been accompanied by a substantial increase in the number of women living post-menopause. It is estimated that many of these women spend more than one-third of their lives in this period, which is marked by a gradual decrease in the production of sex steroids, especially estrogen [1]. Among other consequences, being deprived of estrogen leads to urogenital atrophy, which is associated with progressive, chronic symptoms such as vaginal dryness, vulvar pruritus, a burning sensation in the genitals, sexual symptoms such as dyspareunia and vaginal bleeding [2], along with symptoms related to lower urinary tract dysfunction, such as urinary urgency, increased daily frequency of urination, urinary incontinence (UI), dysuria and recurrent urinary infections [3] [4].

The symptoms stemming from vulvovaginal atrophy and the lower urinary tract are currently referred to as the Genitourinary Syndrome of Menopause (GSM) [5]. GSM, which includes urinary symptoms, can have negative repercussions on the quality of life (QoL), which is why it should always be taken into consideration and addressed so that the patient's aging can be as healthy as possible [4] [6] [7].

Diagnosis of GSM is clinical, based solely on the patient's reported complaints, does not require performing additional examinations, and is confirmed through a careful genital examination, including inspection of the vulva, vestibule, vagina, and urethra in order to detect signs of atrophy, such as dryness, paleness and thinning of the vaginal mucosae. The clinical exam is straightforward and objective and is a useful means of diagnosing GSM, as well as of assessing response to therapy [4].

Since it involves a disorder resulting from the lack of estrogen, GSM requires suitable treatment for recovery of the urogenital epithelium, thus relieving the symptoms and improving QoL. Estrogen replacement is the ideal treatment for GSM and maintains the integrity of the urogenital system. Vaginal administration is the most recommended since it is the best tolerated because, even at low doses, it is effective in restoring vaginal trophism, with little systemic absorption, is safe, and provides rapid improvement in symptoms [8] [9] [10].

The presence of urinary symptoms in GSM is indicative of greater severity of 
the atrophy and correlate directly with lower QoL [11] [12]. Topical estrogen has proven to be effective also for relieving such symptoms and for preventing the recurrent lower urinary tract infections so prevalent in the post-menopausal period since it facilitates the re-colonization of the vagina with lactobacilli, thus inhibiting the pathogens that cause infections [13] [14]. There is also evidence that it is useful in treating irritative symptoms such as urinary urgency and increased day-time frequency [15] [16] [17] [18].

In terms of estrogen therapy, estriol is one of the formulations most often used. Generally, only short-term treatment is needed, for four to twelve weeks, to alleviate the symptoms, and since the doses are low, they can be maintained as long as necessary if there is compliance with all of the safety factors for its use [8] [9].

There is evidence that hormone therapy, in general, can improve health-related QoL in symptomatic women, and it has been assessed using specific questionnaires. In the case of urinary symptoms, QoL assessment has been a fundamental part of the evaluation, whether in clinical practice or surveys. To that end, several questionnaires have been developed, among which is the King's Health Questionnaire (KHQ), developed in the U.K. and translated into and validated for various languages, including Brazilian Portuguese. In addition to identifying lower urinary tract symptoms (LUTS), it also assesses the impact they have on QoL. The International Continence Society (ICS) classified the KHQ as highly recommended or Level A in excellence [19].

The present study was thus developed to assess the effect of the use of vaginal estriol to treat the lower urinary tract symptoms of the Genitourinary Syndrome of Menopause on QoL, using the KHQ questionnaire as the instrument.

\section{Methods}

This study has been conducted in the Hospital Antonio Pedro, at the Universidade Federal Fluminense, in Niterói, Rio de Janeiro State, Brazil. It involved a group of postmenopausal women from the Gynecology outpatient clinics. The study is a clinical, interventional, prospective, individual, longitudinal investigation, with before and after type paired samples. It was performed from August 2014 to April 2015 on that convenience sample, and no previous sample calculation was done.

The inclusion criteria were women presenting with LUTS, who had gone at least one year without menses. The exclusion criteria were patients with contraindications for hormone therapy, or who had a history of deep vein thrombosis, breast cancer, endometrial cancer, renal or hepatic failure, genital prolapse, women who had already used systemic hormone therapy, and those with a cognitive deficit that impeded them to complete the questionnaires.

All of the patients were subjected to anamnesis and a physical examination, and submitted to a Pap test, mammography and transvaginal ultrasound examination. After those procedures, the objectives of the study were explained, and they were invited to take part in it. Those who accepted signed the informed 
consent form and then responded to the KHQ questionnaire. Then each patient was given the estriol vaginal cream and instructed to use one gram, three times a week, for four weeks, followed by the administration of the same dosage twice a week for eight weeks. After treatment, a second assessment of QoL with KHQ questionnaire has been carried on, to be compared with the previous one. The patients themselves completed the questionnaire. If they were unable to do so because of illiteracy or visual deficiency, it was read to them and completed by the lead investigator.

The KHQ questionnaire was translated into and validated for Portuguese by Tamanini et al. in 2003, having been developed to assess effects on the QoL in patients with LUTS. It is comprised of 21 questions that produce a score for the impact of the symptoms on QoL in nine domains: General Health Perception, Incontinence Impact, Role Limitations, Physical Limitations, Social Limitations, Personal Relationships, Emotions, Sleep and Energy, along with Severity Measurements. Numerical values are assigned to all the responses, which are then used to calculate the scores for each domain. Besides, using an independent scale, it identifies the presence and intensity of the following urinary symptoms: increased daytime frequency, nocturia, urgency, urge incontinence, stress incontinence, nocturnal enuresis, intercourse urinary incontinence, frequent urinary infections, bladder pain, and difficulty to pass urine. The higher the score, the worse the quality of life [19].

Data obtained using the KHQ questionnaire constituted the research variables (in nine domains), along with socio-demographic data (age) and clinical data (number of pregnancies and type of delivery, and comorbidities).

The results were reported based on the means and standard deviations for the socio-demographic data and the KHQ domains. Wilcoxon's test was used to compare the scores from the KHQ in two paired samples, before and after the estrogen therapy. The sign test was used to compare the presence of LUTS before and after estriol use. The level of significance adopted was $p<0.05$. The data were recorded on an Excel spreadsheet, and the Statistical Package for Social Sciences (SPSS) program was used for statistical analysis.

The study protocol was approved by the Ethics Committee for Medical Research of the University Hospital Antônio Pedro (Decision no. 826.036).

\section{Results}

Table 1 shows the clinical and sociodemographic characteristics of the 49 women included in the study. Most of the patients (53.6\%) had only vaginal deliveries, and hypertension was the most common comorbidity (61.2\% of the cases). Two women reported deliveries with forceps.

The most frequent LUTS were irritative symptoms (urgency and urge incontinence), referred by $91.8 \%$ of the patients. Difficulty to pass urine was the less common symptom (6.1\%). After estriol, the frequency of all LUTS decreased significantly ( $\mathrm{p} \leq 0.05$ ), except for difficulty to pass urine (Table 2).

The mean scores of the domains of the KHQ questionnaire before and after 
estriol are presented in Table 3. Before treatment, the worst scores were observed for the domains Incontinence Impact $(74.82 \pm 27.66)$ and Severity measures $(48.02 \pm 24.68)$. The less affect domain was Social Limitations (30.38 \pm 28.75).

Table 1. Socio-demographic and clinical characteristics of 49 postmenopausal women attended at the Gynecology outpatient clinics of the Hospital Antonio Pedro, Niterói, Rio de Janeiro State, Brazil.

\begin{tabular}{cc}
\hline Variable & $\mathrm{N}=49$ \\
Age & $64.24 \pm 8.77(46-80)$ yrs. \\
Mean parity & $2.83 \pm 1.86(0-10)$ \\
Mode of delivery & \\
Vaginal & $26(53.1 \%)$ \\
C-section & $12(24.5 \%)$ \\
Both & $9(18.4 \%)$ \\
None & $2(4.1 \%)$ \\
Sexually active & $27(55.1 \%)$ \\
Yes & $22(44.9 \%)$ \\
No & \\
Hypertension & $30(61.2 \%)$ \\
Yes & $19(38.8 \%)$ \\
No & $12(24.5 \%)$ \\
Diabetes mellitus & $37(75.5 \%)$ \\
Yes & \\
No & \\
\hline & \\
\hline
\end{tabular}

Table 2. Frequency of lower urinary tract symptoms reported on the KHQ questionnaire before and after estriol use in 49 postmenopausal women from the Gynecology outpatient clinics of the Hospital Antonio Pedro, Niterói, Rio de Janeiro State, Brazil.

\begin{tabular}{cccc}
\hline & Before estriol & After estriol & p-value $^{\mathrm{a}}$ \\
\hline Urinary symptom & $\mathrm{N}(\%)$ & $\mathrm{N}(\%)$ & $<0.01$ \\
Frequency & $37(75.5)$ & $30(61.2)$ & $<0.01$ \\
Nocturia & $39(79.6)$ & $27(55.1)$ & $<0.01$ \\
Urgency & $45(91.8)$ & $32(65.3)$ & $<0.01$ \\
Stress incontinence & $45(91.8)$ & $33(67.4)$ & $<0.01$ \\
Nocturnal enuresis & $39(79.6)$ & $29(59.2)$ & $<0.01$ \\
Intercourse incontinence & $14(28.6)$ & $6(12.2)$ & $=0.05$ \\
Frequent urinary infections & $10(20.4)$ & $6(12.2)$ & $<0.01$ \\
Bladder pain & $25(51.0)$ & $4(8.2)$ & $<0.01$ \\
Difficulty to pass urine & $21(42.9)$ & $6(12.2)$ & 0.08 \\
\hline
\end{tabular}

a Sign test. 
Table 3. Mean scores of the domains of the KHQ questionnaire in 49 postmenopausal women attended at the Gynecology outpatient clinics of the Hospital Antonio Pedro, Niterói, Rio de Janeiro State, Brazil before and after vaginal estriol use.

\begin{tabular}{cccc}
\hline KHQ domains & $\begin{array}{c}\text { Before } \\
(\text { Means } \pm \text { SD })\end{array}$ & $\begin{array}{c}\text { After } \\
(\text { Means } \pm \text { SD })\end{array}$ & p-value $^{\text {a }}$ \\
\hline General Health Perception & $46.42 \pm 21.65$ & $40.81 \pm 22.64$ & $<0.05$ \\
Incontinence impact & $74.82 \pm 27.66$ & $41.49 \pm 30.83$ & $<0.01$ \\
Role limitations & $43.20 \pm 32.80$ & $21.09 \pm 24.71$ & $<0.01$ \\
Physical limitations & $38.09 \pm 32.09$ & $14.62 \pm 24.20$ & $<0.01$ \\
Social limitations & $30.38 \pm 28.75$ & $12.62 \pm 19.85$ & $<0.01$ \\
Emotions & $43.31 \pm 32.96$ & $20.18 \pm 26.41$ & $<0.01$ \\
Sleep/Energy & $41.48 \pm 37.74$ & $15.98 \pm 23.31$ & $<0.01$ \\
Severity measures & $48.02 \pm 24.68$ & $22.31 \pm 20.25$ & $<0.01$ \\
\hline
\end{tabular}

${ }^{\mathrm{a}}$ Wilcoxon's test. $\mathrm{SD}=$ Standard deviation.

Of the 49 women, only 27 were sexually active, and among them, ten reported coital incontinence. Despite this, even those who were sexually inactive answered that their urinary symptoms did not impact their sex lives. For this lack of consistency in the responses, we decided not to include the domain Personal Relationships in the analysis.

The scores of all analyzed domains decreased significantly after the use of vaginal estriol ( $\mathrm{p} \leq 0.05)$, as presented in Table 3 .

No adverse effects for the use of vaginal estriol were reported.

\section{Discussion}

Assessing health-related quality of life has been the subject of countless studies and publications, mainly starting in the first decade of the 21st century. At a time when technology is overemphasized in Medicine, sustaining and enhancing QoL involves making healthcare more focused on people, and various studies have shown that the QoL of postmenopausal women with LUTS is seriously compromised in several contexts, such as at work, in relationships and their social lives [4] [6] [7]. The literature also suggests that vaginal estriol improves vaginal trophism and reduces LUTS, leading to a positive effect on QoL [9] [17] [18]. In the present study, the comparative analysis showed a decreased frequency of urinary symptoms and average scores in all domains of the KHQ questionnaire after the administration of vaginal estriol, indicating that the use of this hormone had a positive effect on the QoL.

For the General Health Perception domain, it was observed the least difference between the means obtained before and after the use of estriol, although with statistical significance. It is well known that certain age-related disorders occur in the postmenopausal period, such as hypertension, diabetes, emotional disorders, and joint pain, among others. The presence of such comorbidities may lead to a negative impact on QoL and result in the woman having a worse 
general perception of her health. Karakoc et al. showed that GSM occurs more commonly in women who have chronic diseases and use several medications, and in cases of urinary incontinence, which negatively affects their QoL and their ability to take care of themselves [20]. In the present study, a large number of the patients had diabetes and hypertension that, in addition to the use of medications that can cause side effects, may have contributed, along with the LUTS, to lowering their QoL, although no specific analysis was done of that correlation. The improvement in the General Health Perception domain after the use of estriol could be due to improvement in their trophism and urinary symptoms since the patients continued to be subject to their prior comorbidities. Such results are in agreement with the literature [20] [21].

Even though the second domain of KHQ is entitled "Incontinence Impact", it assesses the impact of urinary symptoms in general on QoL, not only incontinence. It showed the highest mean scores in the initial assessment, and presented a statistically significant decrease, demonstrating a subjective improvement in QoL. These results correlate with other studies of the effects of estriol on postmenopausal LUTS, mainly on irritative symptoms such as urinary urgency, frequency, and urge incontinence [17] [18].

Urinary urgency and urge incontinence were the most common symptoms (91\% of cases), followed by stress urinary incontinence and nocturia (79.6\%). It was observed a statistically significant decrease in the frequency of all the urinary symptoms, with improved QOL, which is in agreement with the literature [4] [22]. A decrease in the scores for both the domain Incontinence Impact and in the frequency of urinary symptoms could be justified by the fact that the women had GSM and, after receiving proper treatment, achieved improvement in trophism of their genital and lower urinary tract.

The domain Role Limitations refers to activities such as housecleaning, shopping, or working outside the home and also showed a decrease in the mean scores after the use of vaginal estriol. Many women still work, despite being in their 50's, 60's, or 70's. The effects of LUTS on their productivity have been the subject of several studies, not only from an economic point of view but also due to the embarrassment they cause the patients. Women with UI are less productive and have higher chances of being unemployed than continent women do [23].

LUTS may compromise the ability to take walks, jog, exercise, travel, and have a social life, which may lead to isolation and loneliness, causing depression, which worsens their QoL [6] [24]. In the present study, the decrease in scores in the Physical and Social Limitations domains suggests a positive effect on QoL after the use of estriol, which is in line with other studies that assessed the correlation between UI and social isolation and depression in older women [25].

Negative feelings-such as nervousness, anxiety, and reduced self-esteem, lead to negative effects on QoL. The scores in the Emotions domain of the KHQ suggest that the patients had an improved QoL after using estriol and found their problems alleviated. The studies of Milson et al. and Yip et al. also deemed uri- 
nary urgency to be the leading cause of anxiety and depression [24] [25].

The better scores in the Physical and Social Limitations domains may be explained by the fact that hormone treatment led to better control of micturition, thus providing more confidence in performing actions at home, at work, and in one's social life and leisure pursuits. That effect may also have contributed to better scores in the Emotions domain, by reducing the presence of anxiety due to worries about urinary symptoms during physical and social activities, and thus improving self-esteem.

The substantial negative effect on the Sleep and Energy domain may be related to nocturia, frequently overlooked but highly relevant, because it contributes to a higher rate of falls and higher mortality in the elderly. In the group of 49 women studied, 39 were awakened at night by the desire to urinate, and 14 complained of nocturnal enuresis, showing a high prevalence of those symptoms, with negative effects on QoL since they reported feeling tired and worn out after their sleep cycles were interrupted various times by the desire to urinate. Sleep disorders and lower QoL have been assessed by other studies, with results similar to those found in the present study [22] [26].

In attempting to control their urinary symptoms, many women stop drinking liquids properly or need to use sanitary protective aids, such as pads or diapers. In the Severity Measures domain, women answered how often they had to change their underwear after wetting, and if they worried about the smell of urine, or found their disorder embarrassing. The high scores here suggest a significant negative impact on QoL, which correlates with the study by Tamanini et al., which associates lower QoL with greater severity of the factors assessed [19]. After the use of estriol, there was a significant decrease in the average scores in this domain.

The Personal Relationships domain assesses the extent to which urinary problems affect a woman's relationship with her partner, and her sexual and family life. Of the 49 women, only 27 reported having a sex life, and among them, ten had urine loss during intercourse. Some women may feel uncomfortable discussing sexual matters, but this becomes necessary due to the high frequency of symptoms related to sex life, affecting nearly $50 \%$ of women in the postmenopausal period, negatively affecting their self-esteem and intimacy with their partner, and lowering their QoL [3] [27]. Studies have shown that women who use vaginal estriol have positive effects on their sexual function and QoL [4] [28] [29]. Despite its importance, we decided not to perform a statistical analysis in this domain due to a lack of consistency in the responses we obtained. Only 27 women had sexual intercourse, but even those who were sexually inactive answered that their urinary symptoms did not impact their sex lives. This finding may be due to a limitation of the instrument used, the KHQ, in assessing this domain. Many women avoid intimacy with their partners, not because of urinary symptoms, but due to the vaginal discomfort caused by the atrophy, which may result in pain and bleeding, which, in turn, leads to a loss of desire [29]. Another plausible explanation is that desire declines in women with age, and 
that their lack of sexual activity is not due to the symptoms of GSM, but to other factors.

Our convenience sample and the lack of a control group on placebo are the main limitations of this study. Nevertheless, it reinforces the importance of diagnosing GSM and treating it with vaginal estriol, and of addressing urinary symptoms in the postmenopausal phase, given that patients may be unlikely to report such embarrassing symptoms spontaneously, and that they are potentially under-diagnosed. Healthcare professionals must lay the groundwork for discussing vaginal health and encourage women to talk about this subject, which will make a difference in their QoL, as has been shown in the literature and equally so by the results of this study. Furthermore, the present results suggest that topical vaginal therapy should always be considered when lower urinary tract symptoms are found in women with GSM since it may help improve their QoL.

\section{Conclusion}

The women with postmenopausal lower urinary tract symptoms included in this study had a lower quality of life in all the domains assessed. After using topical vaginal estriol, a positive effect was found on the frequency of urinary symptoms and quality of life, demonstrated by lower average scores in the domains of the KHQ.

\section{Conflicts of Interest}

The authors declare no conflicts of interest regarding the publication of this paper.

\section{References}

[1] Harlow, S.D., Gass, M., Hall, J.E., Lobo, R., Maki, P. and Rebar, R.W. (2012) STRAW+10 Collaborative Group SO. The Journal of Clinical Endocrinology \& Metabolism, 97, 1159-1168. https://doi.org/10.1210/jc.2011-3362

[2] Calleja, A. and Brincat, M.P. (2015) The Urogenital System and the Menopause. Climateric, 18, 18-22. https://doi.org/10.3109/13697137.2015.1078206

[3] Kim, H.K., Kang, S.Y., Chung, Y.J., Kim, J.H. and Kim, M.R. (2015) The Recent Review of the Genitourinary Syndrome of Menopause. Journal of Menopausal Medicine, 21, 65-71. https://doi.org/10.6118/jmm.2015.21.2.65

[4] Nappi, R.E., Martini, S., Cucinella, L., Martella, S., Tiranini, L., Inzoli, A., et al. (2019) Adressing Vulvovaginal Atrophy (VVA)/Genitourinary Syndrome of Menopause (GSM) for Health Aging in Women. Frontiers in Endocrinology, 10, 561. http://www.frontiersin.org/articles/10.3389/fendo.2019.00561/full https://doi.org/10.3389/fendo.2019.00561

[5] Portman, D.J. and Gass, M.L.S. (2014) New Terminology for Vulvovaginal Atrophy from the International Society for the Study of Women's Sexual Health and the North American Menopause Society. Maturitas, 79, 349-354. https://doi.org/10.1016/j.maturitas.2014.07.013

[6] Moral, E., Delgado, J.L., Carmona, F., Caballero, B., Guillán, C., Gonzales, P.M., et 
al. (2018) The Impact of Genitourinary Syndrome of Menopause on Well-Being, Functioning, and Quality of Life in Postmenopausal Women. Menopause: The Journal of the North American Menopause Society, 25, 1418-1423. https://doi.org/10.1097/GME.0000000000001148

[7] Moral, E., Delgado, J.L., Carmona, F., Caballero, B., Guillán C., Gonzales, P.M., et al. (2018) Genitourinary Syndrome of Menopause. Prevalence and Quality of Life in Spanish Postmenopausal Women. The GENISSE Study. Climacteric, 21, 167-173. https://doi.org/10.1080/13697137.2017.1421921

[8] Rahn, D.D., Carberry, C., Sanses, T.V., Mamik, M.M., Ward, R.M., Meriwether, K., et al. (2014) Vaginal Estrogen for Genitourinary Syndrome of Menopause: A Systematic Review. Obstetrics \& Gynecology, 124, 1147-1156.

https://doi.org/10.1097/AOG.0000000000000526

[9] Rueda, C., Osorio, A.M., Avellaneda, A.C., Pinzón, C.E. and Restrepo, O.I. (2017) The Efficacy and Safety of Estriol to Treat Vulvovaginal Atrophy in Postmenopausal Women: A Systematic Literature Review. Climacteric, 20, 231-230. https://doi.org/10.1080/13697137.2017.1329291

[10] Donders, G.G.G., Ruban, K., Bellen, G. and Grinceviciene, S. (2019) Pharmacotherapy for the Treatment of Vaginal Atrophy. Expert Opinion on Pharmacotheraphy, 20, 821-835. https://doi.org/10.1080/14656566.2019.1574752

[11] Crandall, C. (2019) Treatment of Vulvovaginal Atrophy. JAMA, 322, 1910-1911. https://doi.org/10.1001/jama.2019.15100

[12] Nappi, R., Palacios, S., Bruyniks, N., Particco, M. and Panay, N. (2019) The Burden of Vulvovaginal Atrophy on Women's Daily Living: Implications on Quality of Life from a Face-to-Face Real-Life Survey. Menopause, 26, 485-491. https://doi.org/10.1097/GME.0000000000001260

[13] Mody, L. and Juthani-Mehta, M. (2014) Urinary Tract Infections in Older Women. A Clinical Review. JAMA, 311, 844-854. https://doi.org/10.1001/jama.2014.303

[14] Caretto, M., Gianini, A., Russo, E. and Simoncini, T. (2017) Preventing Urinary Tract Infections after Menopause without Antibiotics. Maturitas, 99, 43-46. https://doi.org/10.1016/j.maturitas.2017.02.004

[15] Ostle, Z. (2015) Vaginal Oestrogen for Overactive Bladder in Postmenopausal Women. British Journal of Nursing, 24, 582-585. https://doi.org/10.12968/bjon.2015.24.11.582

[16] Rahn, D.D., Ward, R.M., Sanses, T.V., Carberry, C., Marmik, M.M., Meriwether, K.V., et al. (2015) Vaginal Estrogen Use in Postmenopausal Women with Pelvic Floor Disorders: Systematic Review and Practice Guidelines. International Urogynecology Journal, 26, 3-13. https://doi.org/10.1007/s00192-014-2554-Z

[17] Matarazzo, M.G., Caruso, S., Giunta, G., Valenti, G., Sarpietro, G., Cianci, A., et al. (2018) Does Vaginal Estriol Make Urodynamic Changes in Women with Overactive Bladder Syndrome and Genitourinary Syndrome of Menopause? European Journal of Obstetrics \& Ginecology and Reproductive Biology, 222, 75-79. https://doi.org/10.1016/j.ejogrb.2018.01.002

[18] Weber, M.A., Lim, V., Oryszczyn, J., Te West, N., Souget, J., Jeffery, S., et al. (2017) The Effect of Vaginal Oestriol Cream on Subjective and Objective Symptoms of Stress Urinary Incontinence and Vaginal Atrophy: An International Multi-Centre Pilot Study. Gynecologic Obstetric Investigation, 82, 15-21. https://doi.org/10.1159/000445074

[19] Tamanini, J.T.N., D’Ancona, C.A.L., Botega, N.J. and Netto Jr., N.R. (2013) Validação do "King's Health Questionnaire” para o português em mulheres com incon- 
tinência urinária. Revista de Saúde Pública, 37, 203-211. https://doi.org/10.1590/S0034-89102003000200007

[20] Karakoç, H., Uçtu, A.K. and Ozerdogan, N. (2019) Genitourinary Syndrome of Menopause: Effects on Related Factors, Quality of Life, and Self-Care Power. Menopause Review, 18, 15-22. https://doi.org/10.5114/pm.2019.84152

[21] Bermingham, S.L. and Ashe, J.F. (2012) Systematic Review of the Impact of Urinary Tract Infections on Health-Related Quality of Life. National Guideline Centre, Royal College of Physicians, London, UK. BJU International, 110, 830-836. https://doi.org/10.1111/j.1464-410X.2012.11337.x

[22] Faria, C.A., Menezes, A.M.N., Rodrigues, A.O., Ferreira, A.L.V. and Bolsas, C.N. (2014) Urinary Incontinence and Nocturia: Prevalence and Impact on Quality of Life in Elderly Women in a Primary Health Care Unit. Revista Brasileira de Geriatria e Gerontologia, 17, 17-25. https://doi.org/10.1590/S1809-98232014000100003

[23] Tang, D.H., Cobayco, D.C., Khalaf, K.M., Piercy, J., Patel, V., Globe, D., et al. (2014) Impact of Urinary Incontinence on Healthcare Resource Utilization, Health-Related Quality of Life and Productivity in Patients with Overactive Bladder. BJU International, 113, 484-491. https://doi.org/10.1111/bju.12505

[24] Milson, I., Kaplan, S.A. and Coyne, K.S. (2012) Effect of Bothersome Overactive Bladder Symptoms on Health-Related Quality of Life, Anxiety, Depression and Treatment Seeking in the United States: Results from Epi LUT. Urology, 80, 90-96. https://doi.org/10.1016/j.urology.2012.04.004

[25] Yip, S.O., Dick, M.A., Mc. Pencow, A.M., Martin, D.K., Ciarleglio, M.M. and Ereckson, E.A. (2013) The Association between Urinary and Fecal Incontinence and Social Isolation in Older Women. American Journal of Obstetrics \& Gynecology, 208, 146-147. https://doi.org/10.1016/j.ajog.2012.11.010

[26] Kupelian, V., Fitzgerald, M.P., Kaplan, A.S., Norgaard, J.P., Chiu, G.R. and Rosen, R.C. (2011) Association of Nocturia and Mortality: Results from the Third National Health and Nutrition Examination Survey. Journal of Urology, 185, 571-577. https://doi.org/10.1016/j.juro.2010.09.108

[27] Panay, N. (2015) Genitourinary Syndrome of the Menopause: A Dawn of a New Era? Climateric, 1, 13-17. https://doi.org/10.3109/13697137.2015.1070564

[28] Caruso, S., Cianci, S., Amore, F.F., Ventura, B., Bambili, E., Spadola, S., et al. (2015) Quality of Life and Sexual Function of Naturally Postmenopausal Women on an Ultra Low-Concentration Estriol Vaginal Gel. Menopause: The Journal of the North American Menopause Society, 23, 47-54. https://doi.org/10.1097/GME.0000000000000485

[29] Simon, J., Nappi, R., Kingsberg, S., Maamari, R. and Brown, V. (2014) Clarifying Vaginal Atrophy's Impact on Sex and Relationships (CLOSER) Survey: Emotional and Physical Impact of Vaginal Discomfort on North American Postmenopausal Women and Their Partners. Menopause, 21, 137-142. https://doi.org/10.1097/GME.0b013e318295236f 